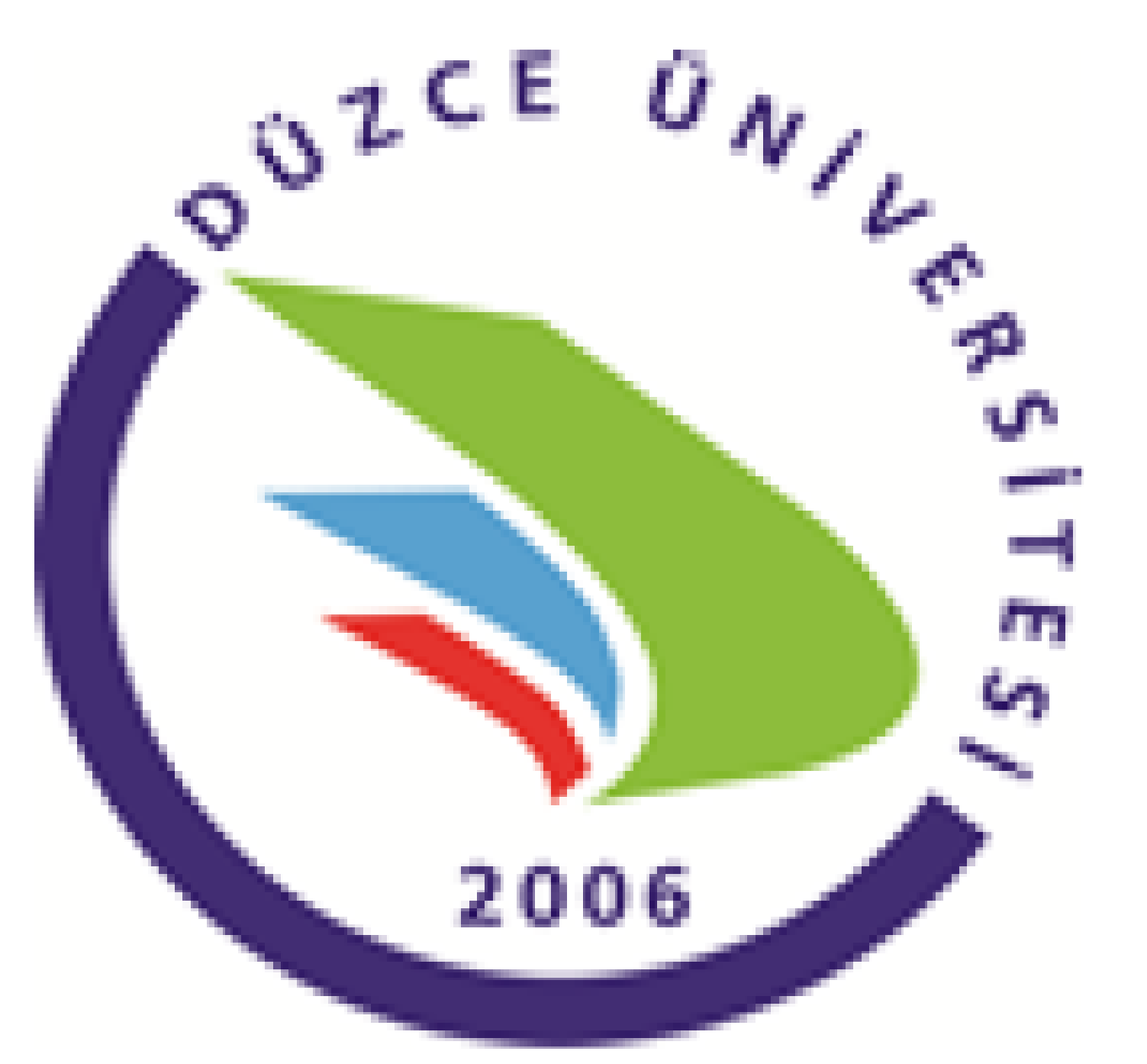

\title{
Anterior Pituitary Hormone Deficiencies in Primary and Surgery Related Empty Sella Cases and Its Effect on Quality of Life
}

\author{
Hafize Titiz, Yusuf Aydin, Elif Onder, Alper Koc, Tuba Soysal
}

Duzce University Medical Faculty Department of Internal Medicine and Endocrinology and Metabolism

Introduction: Primary empty sella (PES) is often found incidentally during neuroradiological studies. Although pituitary function is usually normal in patients with PES, it could be associated with serious clinical conditions including different degrees of hypopituitarism. Postoperative empty sella (POES), the most common group, results from pituitary adenoma and their treatments. Our aim is to compare anterior pituitary functions and quality of life (QoL) with Short Form- 36 (SF-36) between patients with PES (total and partial) and POES.

\begin{tabular}{|c|c|c|c|c|c|c|}
\hline & & & Group 1 & Group 2 & Group 3 & total \\
\hline \multirow{14}{*}{$\begin{array}{l}\text { Hormone } \\
\text { Deficiency }\end{array}$} & \multirow{2}{*}{$\begin{array}{c}\text { No hormone } \\
\text { deficiency }\end{array}$} & Number & 9 & 0 & 3 & 12 \\
\hline & & $\%$ & 26,5 & 0 & 14,3 & 16,0 \\
\hline & \multirow{2}{*}{$\begin{array}{c}\text { Isolated } \\
\text { Gonadotropin } \\
\text { deficiency }\end{array}$} & Number & 5 & 0 & 0 & 5 \\
\hline & & $\%$ & 14,7 & 0 & 0 & 6,7 \\
\hline & \multirow{2}{*}{$\begin{array}{l}\text { Isolated TSH } \\
\text { deficiency }\end{array}$} & Number & 1 & 1 & 1 & 3 \\
\hline & & $\%$ & 2,9 & 5,0 & 4,8 & 4,0 \\
\hline & \multirow{2}{*}{$\begin{array}{c}\text { Isolated } \\
\text { Gonadotropin } \\
\text { deficiency }\end{array}$} & Number & 3 & 2 & 3 & 8 \\
\hline & & $\%$ & 8,8 & 10,0 & 14,3 & 10,7 \\
\hline & \multirow{2}{*}{$\begin{array}{c}\text { GH and } \\
\text { gonadotropin } \\
\text { deficiency }\end{array}$} & Number & 4 & 0 & 2 & 6 \\
\hline & & $\%$ & 11,8 & 0 & 9,5 & 8,0 \\
\hline & \multirow{2}{*}{$\begin{array}{c}\text { GH,TSH and } \\
\text { gonadotropin } \\
\text { deficiency }\end{array}$} & Number & 5 & 2 & 2 & 9 \\
\hline & & $\%$ & 14,7 & 10,0 & 9,5 & 12,0 \\
\hline & \multirow[t]{2}{*}{ Panhypopituitarism } & Number & 7 & 15 & 10 & 32 \\
\hline & & $\%$ & 20,6 & 75,0 & 47,6 & 42,7 \\
\hline & \multirow[t]{2}{*}{ Total } & Number & 34 & 20 & 21 & 75 \\
\hline & & $\%$ & 100,0 & 100,0 & 100,0 & 100 \\
\hline
\end{tabular}

Material and Methods: Seventy-five patients (34 partial, 20 total PES and $21 \mathrm{SES})$ were evaluated. Basal anterior pituitary and its target hormones were measured. When we determined low basal cortisol levels and/or low insulin like growth factor-1 levels, underwent insulin tolerance test (ITT) or synacten test. After the patient gave informed consent to participate in the study, we performed SF-36 to evaluate the QoL.

Table 1: The comparison of pituitary hormone deficiency between patients with PES (total and partial) and POES.

\begin{tabular}{|c|c|c|c|c|c|c|c|}
\hline & $\begin{array}{c}\text { Group 1 } \\
\text { (n:34) }\end{array}$ & $\begin{array}{c}\text { Group 2 } \\
\text { (n:20) }\end{array}$ & $\begin{array}{c}\text { Group 3 } \\
\text { (n:21) }\end{array}$ & p & p1-2 & P1-3 & P2-3 \\
\hline $\begin{array}{c}\text { Physical } \\
\text { functioning }\end{array}$ & $56,2 \pm 26,5$ & $61,2 \pm 27,2$ & $73,2 \pm 26,1$ & 0,068 & & & \\
\hline $\begin{array}{c}\text { Physical role } \\
\text { functioning }\end{array}$ & $22,7 \pm 36,6$ & $35,0 \pm 39,2$ & $61,9 \pm 36,7$ & 0,001 & 0,175 & $<0,001$ & 0,043 \\
\hline Bodily pain & $55,1 \pm 20,9$ & $62,1 \pm 19,0$ & $69,5 \pm 24,9$ & 0,092 & & & \\
\hline $\begin{array}{c}\text { General health } \\
\text { perceptions }\end{array}$ & $29,7 \pm 15,7$ & $42,5 \pm 19,0$ & $48,8 \pm 22,4$ & 0,003 & $\mathbf{0 , 0 1 9}$ & $\mathbf{0 , 0 0 2}$ & 0,480 \\
\hline Vitalite & $31,4 \pm 19,3$ & $37,0 \pm 19,1$ & $45,9 \pm 23,3$ & 0,071 & & & \\
\hline $\begin{array}{c}\text { Social role } \\
\text { functioning }\end{array}$ & $50,2 \pm 23,4$ & $58,2 \pm 24,1$ & $57,8 \pm 23,1$ & 0,369 & & & \\
\hline Emotional role \\
functioning & $33,3 \pm 41,8$ & $33,3 \pm 43,2$ & $49,2 \pm 41,6$ & 0,232 & & & \\
\hline Mental health & $48,1 \pm 17,3$ & $51,1 \pm 17,8$ & $58,7 \pm 17,1$ & 0,086 & & & \\
\hline
\end{tabular}

Results: We classified the patients according to the radiological findings as partial empty sella (Group 1), total empty sella (Group 2) and POES (Group 3). 9 (26,5\%) patients in Group 1 didn't have any hormone deficiency however 15 (75\%) patients from Group 2 and $10(47,6 \%)$ patients from Group 3 and $7(20,6 \%)$ patients from Group 1 had panhypopituitarism The ratio of panhypopituitarism was significantly higher in group 2(Table 1). According to SF-36 scales, Mean score of physical role functioning was significantly higher in Group 3. Score of general health perceptions was significantly lower in Group 1 (Table 2).

Table 2: Mean SF-36 scores according to type of PES (total and partial) and POES

Conclusion: Endocrinological evaluation should be performed during each visit of patients with PES (partial and total) and POES. All hormonal deficiencies should be treated with appropriate medical substitution to maintain the quality of life. QoL in pituitary deficiency were affected in adults, the results showed to physical role functioning and general health perceptions significant difference between groups. 